\title{
Millimetre-wave dielectric spectroscopy for cell analysis
}

\author{
H. Rodilla ${ }^{1}$, A. Kim ${ }^{1,2}$, J. Vukusic ${ }^{1}$, G. D. M. Jeffries ${ }^{1}$, K. Vukusic ${ }^{3}$, A. Jesorka ${ }^{1}$, and J. Stake ${ }^{1}$ \\ ${ }^{1}$ Chalmers University of Technology, Göteborg, Sweden \\ ${ }^{2}$ Karolinska Institutet, Stockholm, Sweden \\ ${ }^{3}$ University of Gothenburg, Göteborg, Sweden
}

\begin{abstract}
A millimeter-wave sensor based on a CPW line has been designed and fabricated as a first prototype for impedance spectroscopy to be combined with a multifunctional micropipette for cell and membrane analysis. The first mm-wave measurement results show the sensitivity of the layout by distinguishing the cells from the media and monitoring the attachment process of the cells to the sensor surface. Measurements were performed on umbilical cord stem cells and cartilage thumb cells.
\end{abstract}

\section{INTRODUCTION}

$\mathrm{M}$ illimetre-wave (mm-wave) and $\mathrm{THz}$ technology has the potential to have a great impact in the fields of biology and medicine [1-3]. Also, microfluidics has emerged as a powerful laboratory toolbox for biologists, allowing manipulation and analysis of processes at a cellular and subcellular level [4].

We present the design of a mm-wave coplanar waveguide (CPW) as a prototype for $\mathrm{THz}$ impedance spectroscopy to be used in combination with a microfluidic multifunctional pipette [5]. This will create a unique setup for multi-, singlecell and cell membrane analysis. The purpose is to be able to monitor cell and membrane activity by detecting impedance changes in the cell and membrane due to internal processes induced by different chemical stimulations applied by the multifunctional pipette. A Schematic illustration of the proposed setup is presented in Fig. 1.

\section{RESULts}

As a first step towards a complete setup including a multifunctional pipette, a mm-wave sensor based on a CPW has been designed in ANSYS HFSS and verified with S-parameters measurements up to $67 \mathrm{GHz}$ with an Agilent Vector Network Analyzer. The CPW design provides flexibility, allowing the study of wide frequency ranges for

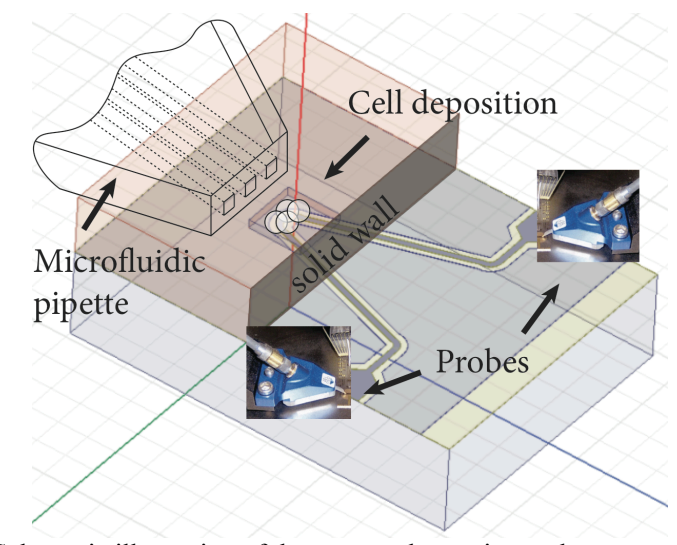

Fig. 1. Schematic illustration of the proposed experimental setup.

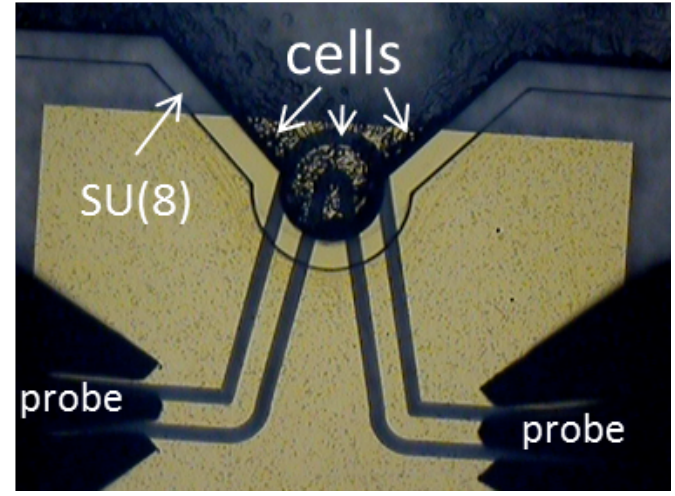

Fig. 2. Image of one of the structures during the experiment with umbilical cord stem cells. Notice that the SU-8 wall isolates the probing area from the cells and culture media.

future experiments. The $\mathrm{Au}$ metallization to form the $\mathrm{CPW}$ was deposited on a glass substrate. Polymer SU-8 walls of 50 $\mu \mathrm{m}$ height were formed on top of the CPW structures in order to isolate the cells and culture media from the probes (Fig. 2). The walls form a closed pool in which the cells immersed in the culture media were placed directly on top of the surface of the sensor. The micropipette will access the cells from the top as a third probe (see Fig.1). During the experiment cells media, umbilical cord stem cells in media and cartilage thumb cells in media where deposited in the pool with a pipette.

In Fig. 3, S-parameter measurements with air, cell culture media and umbilical cord stem cells in the pool are plotted.
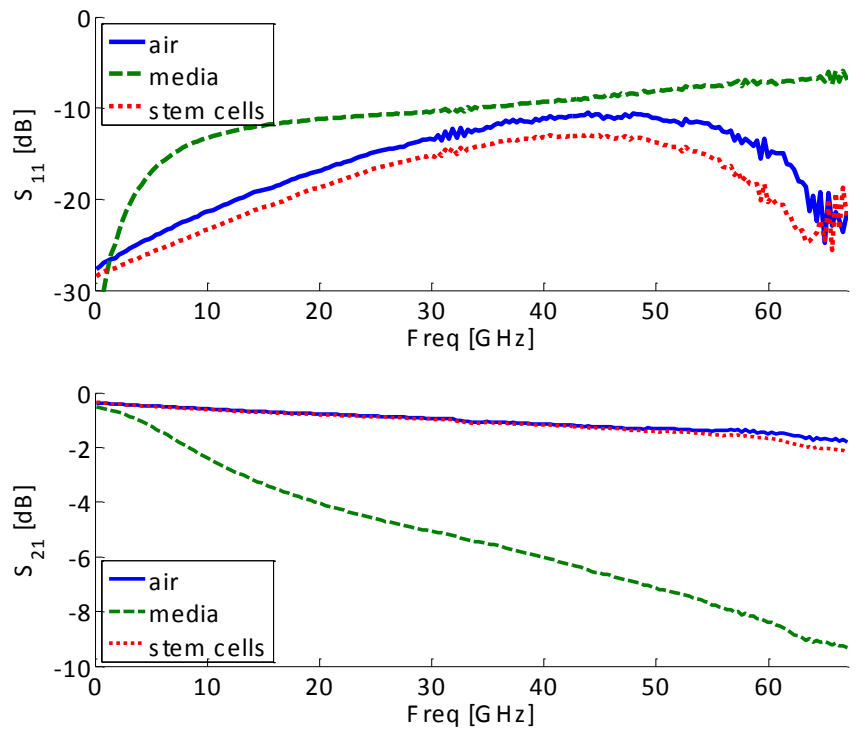

Fig. 3. Experimental results of $S_{11}$ and $S_{21}$ from $100 \mathrm{MHz}$ to $67 \mathrm{GHz}$. Measurements on air (blue continuous line), cell culture media (green dashed lines) and umbilical cord stem cells in culture media (red dotted lines). 

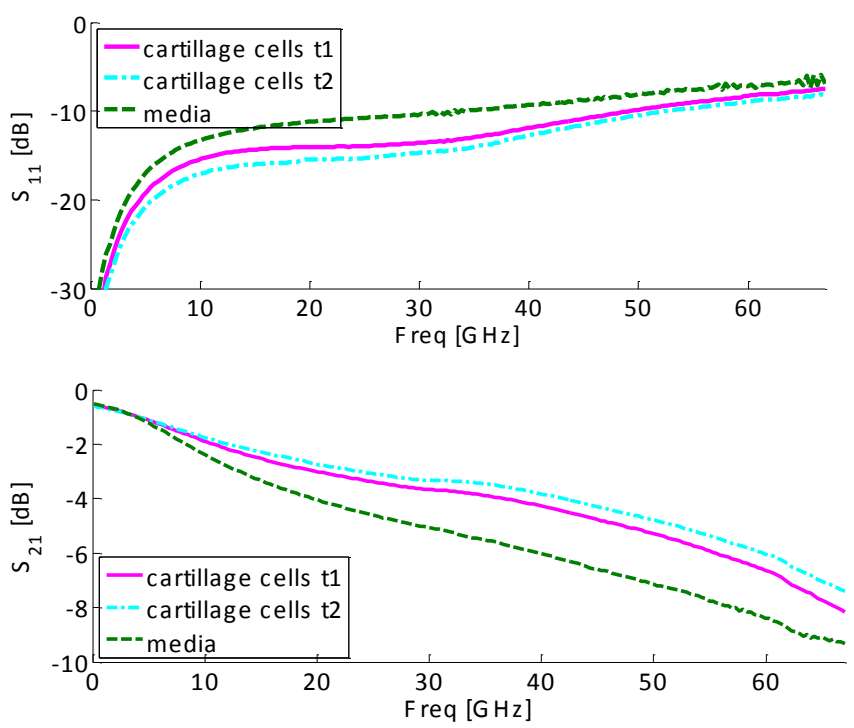

Fig. 4. Experimental results of $S_{11}$ and $S_{21}$ from $100 \mathrm{MHz}$ to $67 \mathrm{GHz}$. Measurements on cell culture media (green dashed lines) and cartilage thumb cells measured at time $t_{1}$ (magenta continuous lines) and $t_{2}$ (cyan dash-dotted lines) with $t_{1}<t_{2}$

The expected increase of the reflectance and absorbance of the cell culture media compared to air has been observed. A clear difference between cell culture media and the umbilical cord stem cells has been observed showing very low losses in the case of the stem cells.

Our sensor is able to record the process of the cells coming into contact with the surface of the sensor. When the droplet with cells and media is deposited in the pool, for a few seconds the measurements are identical to the ones obtained for the media. This is due to the time it takes for the cells to deposit on the surface of the sensor. Once the cells start to deposit onto the sensor's surface, the losses start to decrease continuously with time until the process of attachment to the surface is completed. Control measurements with media have demonstrated that this process is not due to the media evaporation but due to the process of cell addition. An example of this surface adhesion process of the cells is plotted in Fig. 4. Fig. 4 shows $S_{11}$ and $S_{21}$ for cell media and cartilage thumb cells in media. Results for the cartilage thumb cells with two different elapsed times from the drop deposition are represented. $t_{1}<t_{2} . t_{1}$ is an arbitrary time during the process of adhesion and at $t_{2}$ the adhesion process is completed and the cells are attached to the surface of the sensor. As can be

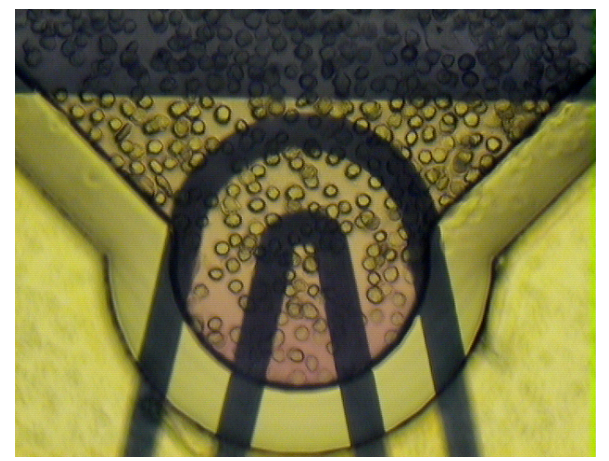

Fig. 5. Image of the sensor during the experiment with cartilage thumb cells in cell culture media.

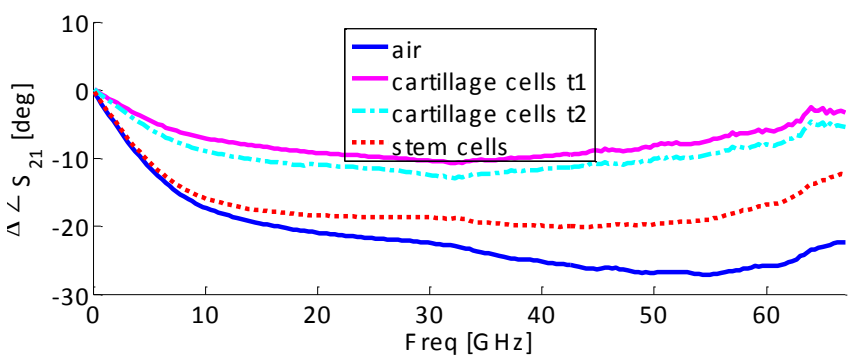

Fig. 6. Phase different in $\mathrm{S} 21$ with respect to cell media of air (blue continuous line), umbilical cord stem cells in culture media (red dotted lines) cartilage thumb cells measured at time $t_{1}$ (magenta solid line) and $t_{2}$ (cyan dash-dotted lines) with $t_{1}<t_{2}$.

observed, the results after a time $t_{l}$ are closer to the ones of the media than the ones after a time $t_{2}$. During the process of attachment to the surface the losses and the reflectance decrease.

Comparing Fig. 3 and Fig. 4 some differences are observed for the two type of cells considered in the experiment. Less losses and reflectance have been observed for the umbilical cord stem cells than for the cartilage thumb cells. We believe the reason is that while the shape of cartilage thumb cells is very round (see Fig. 5) the umbilical cord stem cells are flat (Fig. 2) with larger coverage of the sensor surface.

Fig. 6 shows the difference in phase for $S_{21}$ of air and the two different types of cells considered in the experiment respect to media. The change in permittivity between the air, media, and the two considered types of cells causes not only the change in amplitude observed before in Fig. 3 and Fig. 4, but also change in phase velocity of the wave (Fig. 6).

\section{SUMMARY}

A first prototype of a mm-wave sensor based on a CPW line has been designed for cell impedance spectroscopy analysis. The goal of this sensor is to be combined with a multifunctional pipette to create a unique setup for multi-, single-cell and membrane analysis. Cell and cell membrane activity will be sensed by detecting impedance changes consequence of internal processes induced by chemical stimulations applied by the multifunctional pipette. Our first experimental results show that our structures can distinguish not only between air and media but also clearly identify the cells and indicate a distinction between different levels of adhesion of the cells to the surface of the sensor.

\section{REFERENCES}

[1] P.H. Siegel, "Terahertz Technology in Biology and Medicine,"IEEE Trans. Microw. Theory Techn, vol. 52 pp. 2438-2447, October 2004.

[2] A. Abbas, A. Treizebre, P. Supiot, N.-E. Bourzgui, D. Guillochon, D. Vercaigne-Marko, and B. Bocquet, "Cold plasma functionalized TeraHertz BioMEMS for enzyme reaction analysis," Biosensors and Bioelectronics, vol. 25 , no. 1 , pp. 154-160, Sep. 2009.

[3] S. Laurette, A. Treizebre, A. Elagli, B. Hatirnaz, R. Froidevaux, F. Affouard, L. Duponchel, and B. Bocquet, "Highly sensitive terahertz spectroscopy in microsystem," RSC Adv., vol. 2, no. 26, pp. 10064-10071, 2012.

[4] E. K. Sackmann, A. L. Fulton and D. J. Beebe, "The present and future role of microfluidics in biomedical research," Nature, vol. 507 pp. 181-189, March 2014.

[5] A. Ainla, G. D. M. Jeffries, R. Brune , O. Orwar and A. Jesorka., "A multifunctional pipette" Lab. Chip., vol. 12 pp. 1255-1, January 2012. 